\title{
Simulation Research of Parking Position Aircraft Queuing System Based on Colored Petri Net
}

\author{
Bo Fan, Runtang Wang, Liangcai Cai, Guanhu Wang, Shaofeng Guo \\ Aeronautics Engineering College, Air Force Engineering University. Xi'an, China
}

\begin{abstract}
Keywords: airport engineering; parking position; queuing system; colored Petri net; CPN Tools
Abstract. Form the situation of military aircraft use civil airports, in order to improve the efficiency of the civil airport parking position, this paper gave a new idea that military and civilian aircraft use a shared parking position together, studied capacity and utilization of shared paking position, computed the time military aircraft or civilian aircraft landed the shared parking position. Compared different types of aircraft in and out of the apron to a discrete system, built the colored Petri net queuing system model to calculate the static capacity and the dynamic capacity of shared parking position. Parking queuing system simulation using CPN Tools, to provide theoretical support in study the parking capacity optimization, reduce the wait of queue time, improve the utilization rate.
\end{abstract}

\section{Introduction}

In general, civilian aircraft uses civil airports and military aircraft uses military airports. The military-civilian shared airport is a typical representative of military-civilian integration strategy. The use of civil airports for military aircraft is a problem that needs to be solved in the future integration process. For the study of the queuing system for the parking positions, most of them are currently researching the allocation of parking positions.

The issue of allocation of airport parking positions belongs to the NP-hard combination optimization problem. Domestic airports basically rely on experience to allocate parking positions. Zhao discussed the application of simulation technology in flight area layout evaluation and taxiway system setting[1]. Zhu studied advanced movement guidance and control system based on Petri net theory, some achievements have been made in the field of path planning, conflict detection and liberation[2]; $\mathrm{Xu}$ studied the runway allocation scheme for departing aircraft, and compared the research method with the random allocation scheme, to verify the optimized performance of the method[3]. Yang established a discrete difference model to describe the scene operation, and obtained the relationship between departure taxi time and traffic flow density[4]. The use of computer simulation can further describe the complexity of the airport system. At present, Monte Carlo numerical simulation and discrete simulation techniques are commonly used. The more mature and more elaborate simulation models are SIMMOD and TAAM. This paper uses CPN Tools software based on the colored Petri net theory, simulation study of the queuing system about the parking position.

\section{The theory of Petri net}

Petri net are suitable for describing systems with concurrency and dynamic characteristics, and provide graphical language expressions for system modeling. After many years development, they have produced multiple branches and have been widely used in transportation engineering and aeronautical engineering. The take-off, landing and sliding processes of the aircraft are driven by the activities of the aircraft, and the state of the aircraft is constantly evolving. The process consists of discrete events, and the state of the aircraft changes continuously in each discrete event. Therefore, the operation of the airport is regarded as a hybrid system, formed by the interaction of discrete event dynamic systems and continuous variable dynamic systems[5].

Colored Petri net are further applications of Petri net. They use tokens to represent state identifiers, assign different data types, and refer to data types as token colors. Different types of colors together constitute a color set. Colored Petri net combines Petri net theory with functional programming language Standard ML to model and describe the system. Coloured Petri net is based on the 
separation of tokens in Petri net with different colors in order to realize the folding and simplification of the net system[6].

The mathematical language of the colored Petri net is described as[7]:

$$
C P N=\left(\sum, P, T, F, N, C, G, I, E\right) \text {. }
$$

$\sum:$ the color set; $P$ : the place set; $T:$ the transition set; $F:$ the directed arc; $N:$ the node function; $C$ : color function mapped from 1 to $2 ; G$ : Alert function,

$\forall t \in T:[\operatorname{Type}(G(t))=\{$ true, false $\} \wedge \operatorname{Type}(\operatorname{Var}(E(a))) \in \Sigma]$,

Type: type function; Var: variable function;

$I$ : Initialize the function, define the initial mark in the place, $\forall p \in P:\left[\operatorname{Type}(I(p))=C(p)_{M S}\right]$;

$E$ : Arc function, $\forall a \in A:\left[\operatorname{Type}(E(a))=C(p)_{M S} \wedge \operatorname{Type}(\operatorname{Var}(E(a))) \in \sum\right]$.

As a high-level Petri net model, the colored Petri net has different characteristics and rules in its structure, labeling, and excitation[8]. Details as follows:

(I) Declaration and labeling

The place is represented by an ellipse. There are two labels around it, which respectively represent the color set and initial state of the place. The definition of the initial state can be described as a polymorphic set consisting of multiple tokens. When the transition is triggered, the token is changed with the input place transferred to the output place. The color type of the transferred token is determined by the arc expression. The color set and arc expression are defined by the CPN ML beforehand by defining the variables, constants, and functions. Places and transitions can be given different names to express their meaning in the actual system.

\section{(II) Excitation of transitions}

The excitation of the transition needs to satisfy the conditions corresponding to the types of variables in the arc expression and its input place polymorphism set (set of different types, quantity tokens). At the same time, the arc expression also limits the multi-state set that can be transferred in the input place. The token under this condition is called binding. The change trigger also needs to satisfy the boolean value of the alert function as true.

(III) Excitation and execution steps

When there are two bindings to be transferred in one input place of the transition, and there is only one token in the other input place, the two bindings are in a conflicting relationship. When there are enough tokens in other input places, there is a concurrency relationship. The continuous execution process of the CPN model is regarded as a transition excitation sequence, and at the same time, the identification reachability sequence of the model execution can also be obtained.

(IV) Hierarchical Model

The CPN model represents a CPN submodule with alternative transitions. The input/output places for substitution transitions can be mapped to the input/output places of submodules. The CPN model can fuse places that represent the same meaning and form a converged place.

(V) Time

The CPN model represents the operation of a real-time system by adding time information. The time information is divided into two types. A timestamp is added after the token to indicate the time status information of the polymorphic set in the place. Another type of time information is global time, which represents the total time the model was running. After the time information is given, the order in which the tokens are excitationed is determined by the time stamp and the global time. The changing code segment function and delay function can calculate the time required for the state transition, the time when the delay occurs, and assign the time information to the token through an arc expression.

(VI) Model of aircraft ground operation

In the ground operation model of the aircraft, ground moving objects are represented by tokens. The state of the system is described by the token in the venue. If the place is empty, it means that there is no active object in the zone at the moment. If the place contains a token, it means that the active object stays in this zone or is passing through this zone. Fig.1 shows a basic aircraft ground operation model. When the aircraft sliding from active zone 1 to active zone 2, the corresponding token is transferred 
from place $P_{1}$ to place $P_{2}$ in the colored Petri net model. The process of the aircraft crossing the boundary line is represented by the excitation of transition $t_{1}$.

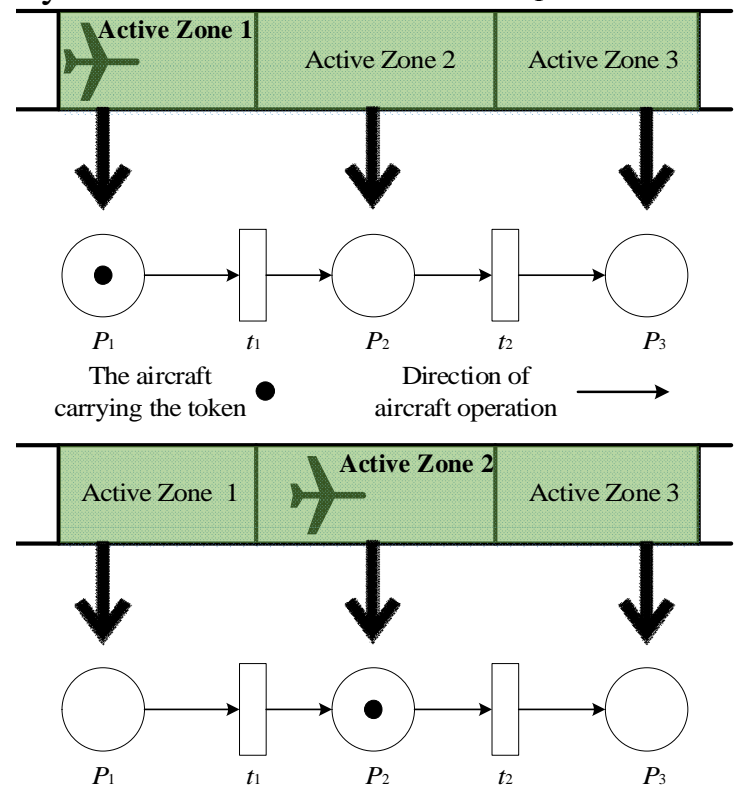

Fig.1 A basic model of an aircraft operating on the ground

\section{Calculation model of apron capacity}

According to the function of the flight area, airport capacity can be divided into runway capacity, taxiway capacity, and apron capacity. In general, the capacity of the taxiway beyond the runway capacity and apron capacity is not a bottleneck that limits the capacity of the entire airport. Runway capacity is a major aspect of military airport capacity constraints. The apron capacity is sometimes the limiting factor for the entire airside of a small landing airport, especially for the use of civil feeder airports for military aircraft, where the capacity of the apron limits the capacity of the entire airside[9].

The apron is divided into a collective apron and individual apron. The civil airport is generally a collective apron, which is convenient to use and has a high utilization rate. The apron of civil aviation is located on the airside and serves as a connection between the terminal area building and the flight area. It is used for aircraft parking and the passengers up and down. There are bridge gate position and a far-away parking position, military aircraft mainly use the far-away parking positon of civil airports and try to stay away from the terminal area. The current mode of operation of military aircraft using civil airports is that the apron area of military and civilian aircraft is completely independent. This paper proposes to divide the apron of the civil airport into a civilian aircraft apron, a military aircraft apron, and a military-civilian shared parking apron, as shown in Fig.2.

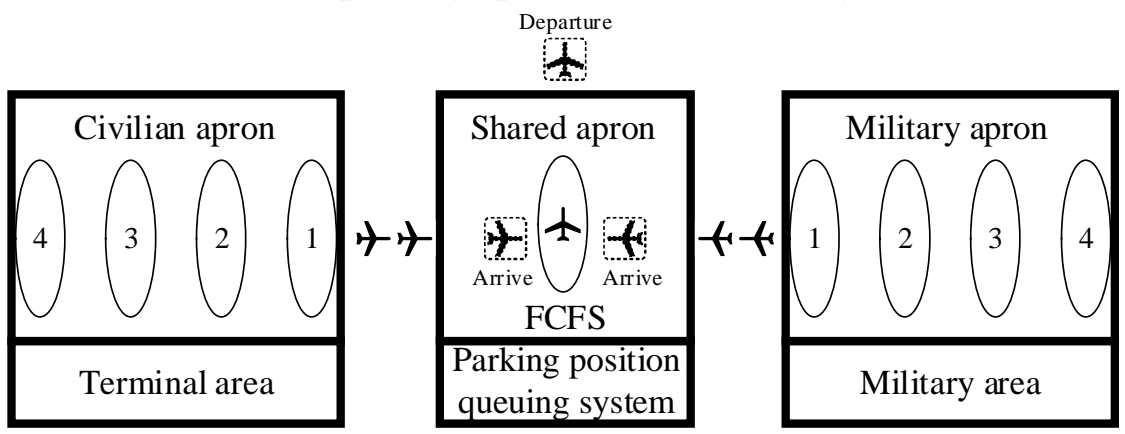

Fig.2 Area division of apron

Whether it is a civilian aircraft apron, a military aircraft apron or a civilian-military shared apron, the effective index of apron capacity is determined by the number of parking positions $N$. It is called static capacity, refers to the maximum number that aircraft can occupy the apron at any given moment. The static capacity of the parking position is expressed as: 


$$
C_{\text {static }}=N
$$

Static capacity often provides instantaneous values of apron capacity that are not easily comparable to runway capacity or capacity of the other parts[10]. The dynamic capacity of the apron, as a widely used measure, refers to the number of positions accepted by the aircraft per hour, which is in line with the concept of runway capacity.

(I) SOT: scheduled occupancy time, under normal circumstances the scope of change is $20 \mathrm{~min} \sim 4 \mathrm{~h}$, SOT for regional routes is $20 \mathrm{~min}$, SOT for the wide-body aircraft on international routes is $4 \mathrm{~h}$, does not include overnight up to $10 \mathrm{~h} \sim 12 \mathrm{~h}$ occupancy time.

(II) BT: flight position buffer time, which determines the average interval between consecutive flight positions. The actual buffer time depends largely on the situation of the local airport. The range is generally 5 minutes to 1 hour or even longer.

(III) PT: the placement time, which is generally 2 to 4 minutes for the far-away position and 10 to 30 minutes for the near position.

According to the appropriate combination of criteria, including the size of the aircrafts or airlines, the arriving aircraft is divided into $K$ types. For each type $i$ of aircrafts, the aircraft scheduled blockade time $S B T$ is the sum of $S O T, B T$, and $P T$. The classification of aircrafts for calculating the apron capacity does not have to be the same as the model classification for runway capacity calculation[11]. $p_{i}$ is the percentage of $i$ aircraft arriving at the airport. Calculate the expectation:

$$
E[S B T]=\sum_{i=1}^{K} p_{i} \times S B T_{i}
$$

The dynamic capacity of the parking position is expressed as:

$$
C_{\text {dynamic }}=\frac{N^{2}}{E[S B T]}
$$

\section{Simulation of CPN Tools}

CPN Tools is developed by Aarhus University, which is mainly used for the modeling of colored Petri net for discrete or concurrent systems, and analyzes the performance of the system through simulation. Fig. 3 is the user interface of CPN Tools, which consists of index area and workspace. The index area mainly includes toolbox, variable declaration and function definition, structure and operation of CPN model. After we drag the tool on the right side of the workspace, expressed in a dialog box, every tool contains many functions, including model creating, state space tool, hierarchical model construction and simulation, etc. The right workspace (the new page dialog in the diagram) builds and operates a live page for the model, which can represent a large amount of information through modeling.

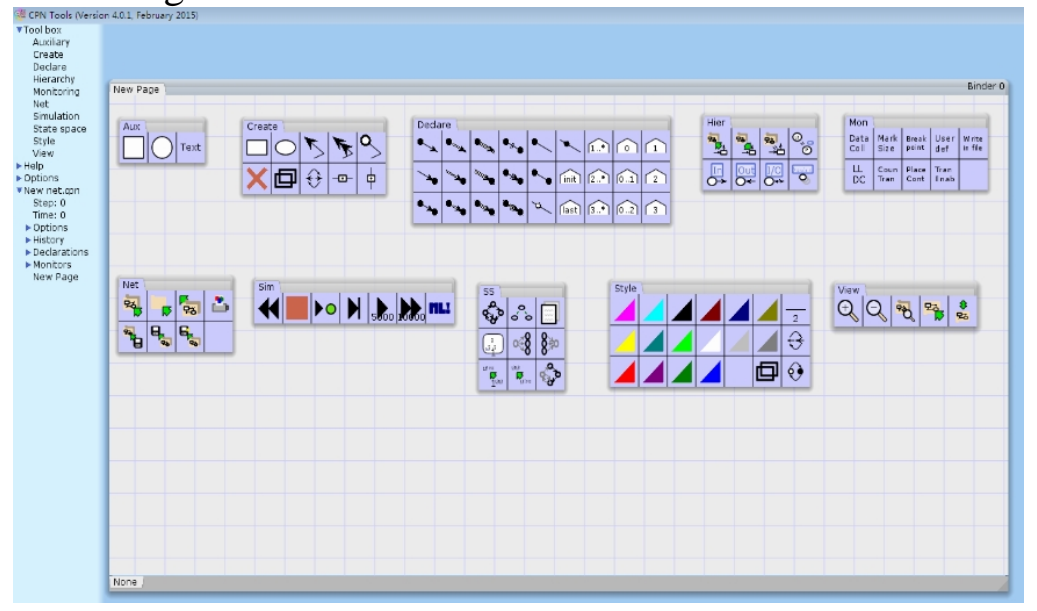

Fig.3 User interface of CPN Tools 
According to the characteristics and process of an aircraft uses parking position, the algorithm steps of constructing the colored Petri net model of the aircraft in the shared parking position queuing system are as follows[12]:

Step 1: each independent aircraft activity is represented by a transition;

Step 2: if transition $t_{i}$ is a tight front activity of $t_{j}$, add a place $p$ between $t_{i}$ and $t_{j}$, connect two arcs from $t_{i}$ to $p$ and from $p$ to $t_{j}$;

Step 3: if there is no pre-sequence activity of $t_{b}$, add a place $p_{b}$ in front of $t_{b}$, connect an arc $p_{b}$ to $t_{b}$, and put a token in $p_{b}$;

Step 4: if there is no post-sequence activity of $t_{\mathrm{e}}$, add a place $p_{\mathrm{e}}$ behind $t_{\mathrm{e}}$, connect an arc $t_{\mathrm{e}}$ to $p_{\mathrm{e}}$; Step 5: assign time to transitions of all aircraft activities.

\section{Simulation of parking position queuing system}

This paper discretizes the process of aircraft in and out of the parking position. Using the excitation of transition to indicate the change of aircraft state. Invoking the transition function and arc function, to calculate and return the interval and duration time of the aircraft at each state. A token is used to represent an aircraft, and the flow of tokens indicates the operation of the aircraft in various processes. In order to simplify the structure of the network, the CPN Tools simulation model was used to establish the colored Petri net model. Petri net model of aircrafts queuing system, as shown in Fig.4.

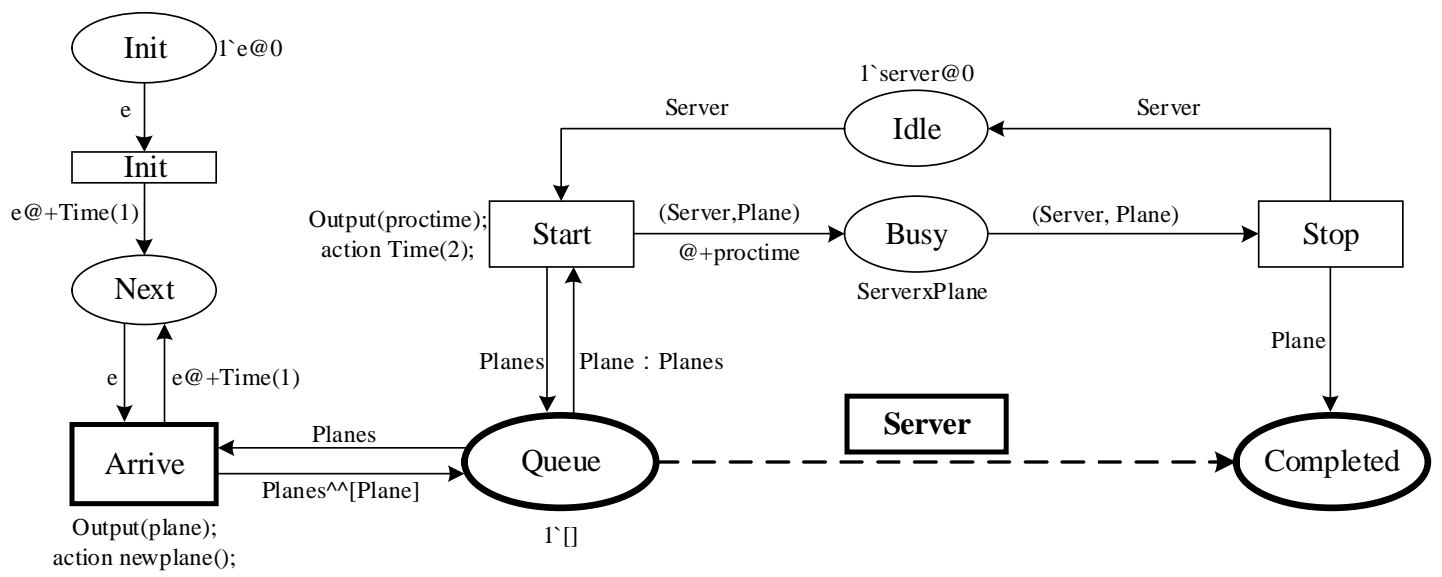

Fig.4 Petri net model of the parking position queuing system

Assuming that each position of the parking apron can only stay on a military aircraft or a civilian aircraft, another aircraft will continue to use the parking position after the end of the flight.

The simulation model is expressed as follows: in the parking position queuing system, the shared parking position is represented by the Server. The color set PlaneType defines two types of aircraft: A and $\mathrm{B}$, respectively representing military aircraft and civilian aircraft. The place Idle and the place Busy are used to represent the state of Server. Place Idle represents idle state and there does not have a parking position, Place Busy represents busy state and there has an aircraft. The time function of aircraft arriving at the parking position is expressed with Time(1). The time function of aircraft is parked in the parking position with Time(2). ServerxPlane is the color set that is used to represent the Server when the Server is busy processing the job.

After place Init on the queuing system initialization, the aircraft which using shared parking position arrived with function Time(1), if the aircraft arrived Server is busy, will be added to the queue at the end of the aircraft. The queue strategy is first come, first served, that is FCFS. If the Server is idle when the aircraft arrives, the aircraft immediately passes the empty queue and uses the parking position. When the aircraft departs the parking position, the Server becomes idle. If the parking position is idle, the aircraft in the queue is waiting, and the aircraft in front of the queue first uses the parking position.

Finally, useing the Monitor function of CPN Tools to write a program to calculate the following performance indicators of the queuing system: Queue Length, Queue Delay and Server Utilization. 
After setting the number of operations, the simulation results were in the corresponding Output folder.

\section{Conclusions}

Military aircraft use civil airports, the far-away parking position of the civil airport is used as a shared parking position for military and civil aviation. Optimize the dynamic capacity of the position and improve the utilization rate of the parking position. Taking Xinjiang Kashgar Airport of China as an example, although it is a civil airport, has been used by military aircraft training camp for years, and its military position and civil position are completely independent. There are two E class far-away parking positions and six D class bridge gate position in the civil apron, but the utilization rate of the far-away parking positions is very low, and the military position is basically saturated. In this paper, through the establishment of shared parking position coloured Petri net model, application of CPN Tools simulation queuing system, provides a new method for the feasibility of using the two far-away parking positions as the shared parking positions for the military and civil aviation. It will also promote the integration of military and civilian development.

\section{Acknowledgements}

This work was financially supported by the National Natural Science Foundation of China (51578540).

\section{References}

[1] ZHAO H D, LING J M, ZHANG P. Evaluation Method of Airport Flight Layout Based on Discrete Simulation Analysis[J]. Journal of Civil Aviation University of China, 2013,31 (06): 21-25. [2] ZHU X P. Research on The Taxi Route and Conflict Monitoring Theory of A-SMGCS Aircraft Based on Petri Net[D]. Nanjing: Nanjing University of Aeronautics and Astronautics, 2012.

[3] XU T, HU M H, ZHAO Z. Simulation Model and Algorithm for Multi-track Departure Field Assignment[J]. Aviation Computing Technology, 2015,45 (01): 91-95.

[4] YANG L, HU M H, YIN W S, et al. Analysis of Traffic Flow Congestion in the Scene of Large-scale Busy Airport Scene[J]. Journal of Aviation, 2016,37 (06): 1921-1930.

[5] GAO C. Study on the Stability of Hybrid Systems[D]. Zhenjiang: Jiangsu University, 2012.

[6] MA Holliday, MK Vernon. A Generalized Model for Performance Analysis[J]. IEEE Transactions on Software Engineering, 2006, SE-13(12): 1297-1310.

[7] ZHANG Z Q. A Study on the Analysis and Verification of Modeling and Model Properties Based on Pigmentation Petri Net[D]. Jinan: Shandong university, 2012.

[8] BAO J, LU T. Modeling and Simulation of Situational Awareness System Based on Petri Net[J]. Computer Application Research, 2012, 29(2) : 621-625.

[9] FAN B, CAI L C, WANG G H, et al. A Calculation Method of Runway Length Safety Coefficient Based on Aircraft Landing Safety[J]. Journal of Air Force Engineering University (Natural Science Edition), 2018, 19(2): 9-14.

[10] Richard DE Neverwell, Armitage R. O'donnell, GAO J H, et al. Airport Systems: Planning, Design and Management[M]. Beijing: China Civil Aviation Press, 2006.

[11] ZHU J F. Aviation Transportation Planning[M]. Xi 'an: Northwest University of Technology Press, 2009.

[12] TANG X M, ZHU X P. PETRI Net Theory and It's Application in Civil Aviation Transportation Engineering[M]. Beijing: China Civil Aviation Press, 2013. 